\title{
Estudio anual del zooplancton: composición, abundancia, biomasa e hidrología del norte de Quintana Roo, mar Caribe de México
}

\author{
Annual study of zooplankton: composition, abundance, biomass and hydrology from the north \\ of Quintana Roo, Mexican Caribbean Sea
}

\author{
José N. Álvarez-Cadena1* ${ }^{*}$, Uriel Ordóñez-López ${ }^{2}$, David Valdés-Lozano², Alma Rosa Almaral-Mendívil ${ }^{1}$ y \\ Amira Uicab-Sabido ${ }^{2}$ \\ ${ }^{1}$ Universidad Nacional Autónoma de México. Instituto de Ciencias del Mar y Limnología, Unidad Académica Puerto Morelos. Apartado postal 1152, \\ 77501, Cancún, Quintana Roo, México. \\ ${ }^{2}$ Instituto Politécnico Nacional, Centro de Investigación y Estudios Avanzados, Unidad Mérida, Km. 6 Antigua carretera a Progreso. A.P. 73 \\ CORDEMEX, 97310, Mérida, Yucatán, México. \\ *Correspondencia: inac@mar.icmyl.unam.mx
}

\begin{abstract}
Resumen. Se llevaron a cabo muestreos de zooplancton en la zona lagunar y costera del Caribe mexicano, desde Puerto Morelos hasta Cancún. Las recolectas se llevaron a cabo de enero a diciembre de 2004 en 12 localidades. Se identificaron 41 grupos del zooplancton donde los copépodos fueron los más abundantes (61\%) seguidos de las larvas de equinodermos (17\%) y decápodos (5\%). El copépodo Acartia tonsa fue la especie más abundante de este grupo en el Sistema Lagunar Nichupté (SLN). En la zona marina adyacente los copépodos estuvieron representados en orden de importancia por Acartia spinata, Pseudocalanus sp. y Calanopia americana. En todas las estaciones se capturaron equinodermos del tipo equinopluteus-ofiopluteus, pero con mayor abundancia en el SLN. El quetognato Ferosagitta hispida fue la única especie que se encontró en el SLN, donde fue más abundante. Los decápodos estuvieron representados principalmente por larvas zoeas; las larvas de peces por 54 familias, de las cuales los góbidos de los géneros Ctenogobius sp., Gobionellus sp. y Gobiosoma sp. fueron los mejor representados, particularmente para el SLN. La biomasa fue mayor en el SLN.
\end{abstract}

Palabras clave: zooplancton, Caribe mexicano

\begin{abstract}
Zooplankton sampling was carried out in the northern coast of the Mexican Caribbean Sea, from Puerto Morelos to Cancun. Captures were made with a conic net $0.4 \mathrm{~m}$ diameter, $1.40 \mathrm{~m}$ length and $0.330 \mathrm{~mm}$ mesh from January to December 2004 at twelve locations. A total of 41 zooplankton groups were identified. Copepods were the most abundant taxa making up 61\%, followed by echinoderms (17\%) and decapods (5\%). Acartia tonsa at the Nichupte Lagoon System (SLN) over numbered the copepod fauna and occasionally the whole zooplankton population. Along the coast Acartia spinata, Pseudocalanus sp, and Calanopia americana were the most important copepods. Echinoderms larvae such as echinopluteus-ophiopluteus were present at all sampled stations but were more abundant at the NLS. For chaetognaths, Ferosagitta hispida dominated, the species was the most abundant and in fact the only species found within the NLS. Decapod larvae were mainly represented by zoea larvae (brachiura). Fish larvae were made up by 54 families, from those, Gobids of the genera Ctenogobius sp., Gobionellus sp. and Gobiosoma sp. were the most abundant, particularly for SLN. Biomass was higher at stations located within the SLN.
\end{abstract}

Key words: zooplankton, Mexican Caribbean Sea.

\section{Introducción}

Los ambientes arrecifales son lugares de alta productividad rodeados de mares con condiciones oligotróficas de bajo contenido en nutrientes. Los

Recibido: 24 octubre 2006; aceptado: 25 abril 2007 arrecifes coralinos, de acuerdo con Alldredge y King (1977) y Sorokin (1978), deben considerarse de alta riqueza planctónica debido a la abundante presencia de organismos que se alimentan por filtración. El Caribe de México, pese a su gran importancia, es una de las áreas que menos se han estudiado, particularmente en lo que a plancton se refiere, y otros mares patrimoniales del país 
tienen más información respecto del estado que guardan sus aguas costeras o de mar abierto. Para la parte norte del Caribe de México, la información más relevante respecto a las comunidades del plancton se puede encontrar en los trabajos de Suárez-Morales y Gasca (1990) (zooplancton), Álvarez-Cadena y Segura-Puertas (1997) (zooplancton) Álvarez-Cadena et al. (1998) (Copepoda), Ramírez- Ávila y Álvarez-Cadena (1999) (Chaetognatha), Ramos-Viera (2001) (Medusae), Muñoz-Salcedo (2005) (Medusae), $\mathrm{y}$ estudios relacionados con el ictioplancton han sido llevados a cabo por Ramírez-Ávila (2001) y por OrdóñezLópez (en prensa).

En este trabajo se aborda por primera vez el estudio de los grupos de zooplancton en la zona norte del estado de Quintana Roo, en el lapso de un año, y relacionando su composición y abundancia con las variaciones de los parámetros hidrológicos.

\section{Material y métodos}

El área de estudio forma parte de la segunda barrera arrecifal más grande del mundo, sólo después de la gran barrera coralina de Australia. Se inicia en las inmediaciones de Cancún y se extiende hasta Belice. Los lugares de muestreo se ubican en la parte noreste de la península de Yucatán $\left(20^{\circ} 49^{\prime}\right.$ a $21^{\circ} 10^{\prime} \mathrm{N}$; $86^{\circ} 43^{\prime}$ a $86^{\circ}$ $55^{\prime} \mathrm{O}$ ) desde Puerto Morelos hasta Cancún, incluyendo el Sistema Lagunar Nichupté (SLN) este último localizado en la vecindad de la zona hotelera (Fig.1).

De acuerdo con Merino (1986), las corrientes oceánicas en este lugar tienen un flujo con dirección norte, las cuales se continúan y eventualmente forman parte de la corriente de Yucatán hasta alcanzar el golfo de México. Así también se han registrado contracorrientes de menor intensidad. Los vientos alisios (sur-sureste) son dominantes durante la mayor época del año, excepto durante el invierno cuando se registran fuertes vientos del norte. El fondo esta típicamente cubierto de vegetación sumergida conformada por pastos marinos (Thalassia testudinum Banks ex König y Halodule sp.) intercalados con blanquizales de arena (en el SLN se registran también sedimentos lodosos). El régimen de mareas es semidiurno con un corto rango de variación (0.24m) (Instituto de Geofísica, 1991).

Se llevaron a cabo muestreos mensuales de zooplancton de enero a diciembre de 2004 en 12 localidades de la zona lagunar y costera del norte de Quintana Roo, de Puerto Morelos a Cancún, en el Caribe de México (Fig.1). Las capturas se hicieron mediante una red tipo cono truncado de $0.40 \mathrm{~m}$ de boca y $1.4 \mathrm{~m}$ de longitud (malla de 0.330 $\mathrm{mm}$ ) por $5 \mathrm{~min}$., provista de un medidor de flujo para hacer estimaciones de densidad de organismos por $100 \mathrm{~m}^{3}$.

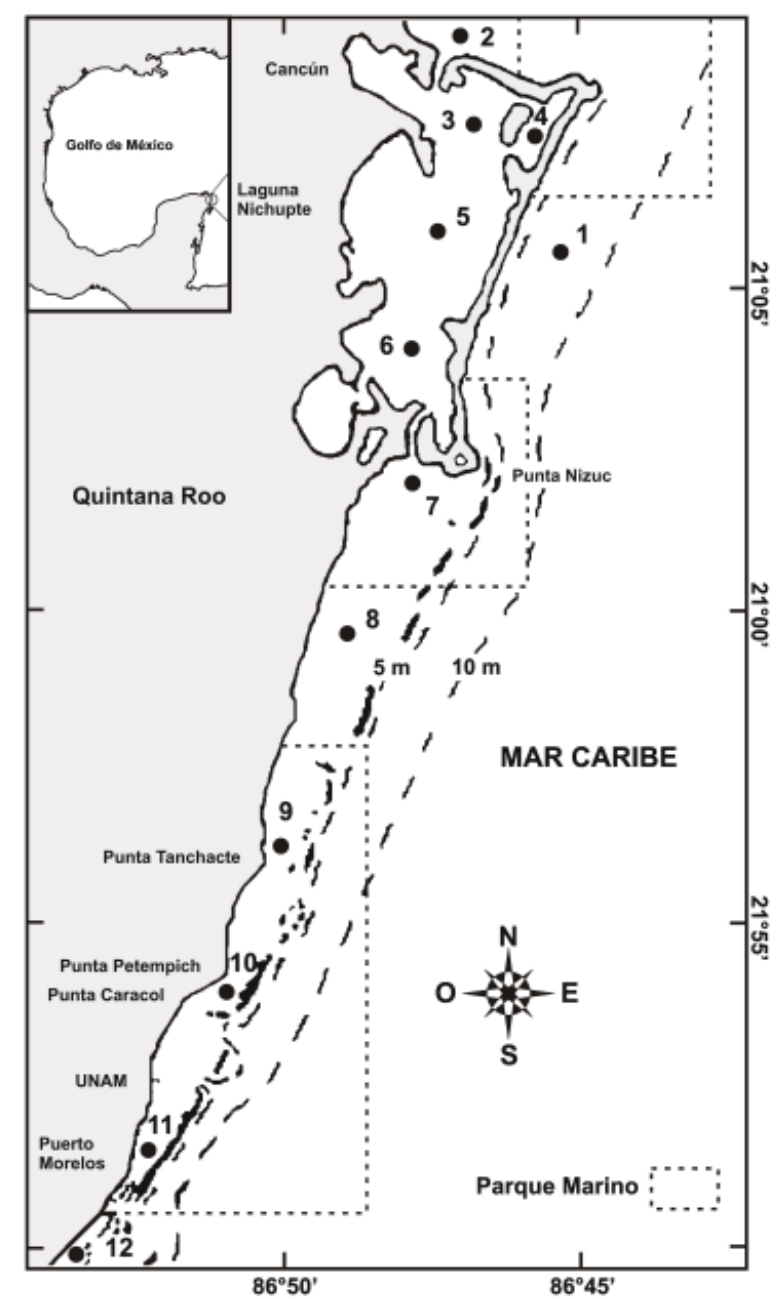

Figura 1. Ubicación de los lugares de muestreo (1-12) en la zona lagunar y costera del norte de Quintana Roo, México.

Las muestras obtenidas se fijaron inmediatamente después de su captura con formol a una concentración de $4 \%$ amortiguado con borato de sodio. Los parámetros hidrológicos superficiales de temperatura $\left({ }^{\circ} \mathrm{C}\right)$, salinidad (en ups, unidades prácticas de salinidad) y oxígeno $(\mathrm{ml} /$ 1) se midieron in situ con un multisensor de campo YSI$85( \pm 0.1)$. Asimismo, se obtuvo un litro de agua para el análisis de clorofilas por el método espectrofotométrico descrito por Stricklan y Parson (1972). La determinación de biomasa se realizó por el método de peso húmedo de acuerdo a Beers (1981). En todos los casos se cuantificaron cuando menos 800 organismos por muestra, alícuota que fue considerada representativa de la muestra total de acuerdo a los criterios de Harris et al. (2000).

A los datos hidrológicos espaciales y temporales obtenidos se les aplicó un análisis de clasificación aglomerativa mediante el coeficiente de Bray-Curtis 
a fin de identificar grupos de estaciones y/o meses, y se sometieron al análisis de varianza (ANOVA) de 2 vías $(\mathrm{p}<0.05$, Zar, 1988) para conocer si existen diferencias significativas respecto a una misma variable. Este procedimiento se repitió con la abundancia de los grupos del zooplancton. Finalmente, se efectuó un análisis de correspondencia canónica (ACC) con el fin de conocer las posibles relaciones entre los grupos dominantes y los parámetros ambientales registrados. Este procedimiento permite posicionar los grupos de organismos con los sitios y los factores ambientales en un sistema sintético de coordenadas (componentes 1 y 2) (Ter Braak y Verdonschot,1995).

\section{Resultados}

El análisis de agrupación de las estaciones y/o meses, basado en los promedios de las principales variables superficiales del medio permitió identificar un gradiente hidrológico espacial (por zonas) y otro temporal (porépocas) en la zona costera norte de Quintana Roo. Espacialmente fueron 3 zonas, la costera (con características oceánicas, estaciones 1 y 2), la lagunar (SLN, estaciones 3-6) y la arrecifal (con influencia de la barrera coralina, estaciones 7-12), y temporalmente, la época de sequía de noviembre a mayo y la de lluvias de junio a octubre.

Cada uno de estos grupos fue diferente respecto a los otros de manera significativa (ANOVA ${ }_{\mathrm{F} 2,234=3.69, \mathrm{P} \leq 0.05}$ ), en términos de las variables abióticas y bióticas del medio (Cuadro 1).

La mayor temperatura promedio del agua se localizó en las estaciones relacionadas con el medio arrecifal y en el SLN (28.4 y $28.2^{\circ} \mathrm{C}$, respectivamente). Es probable que estos valores, en el primer caso, estén relacionados con la temperatura acumulada durante el transcurso del día y en el segundo, con lo somero del cuerpo de agua del SLN, principalmente durante la época cálida.

Lo anterior también influyó en un aumento de la salinidad en el medio arrecifal (36.1 ups), sobre todo durante el estiaje. En el SLN, la menor salinidad (30.4) se debió a importantes aportes de agua dulce de procedencia continental ya sea por escurrimientos o por afloramientos de aguas subterráneas y que fueron más abundantes durante la época de mayor precipitación pluvial, lo cual ha sido registrado en trabajos previos (Merino et al.,1990; Boa-González, 1993).

Las concentraciones de oxígeno superficial fueron mayores en el medio arrecifal $(5.4 \mathrm{mg} / \mathrm{l})$ que posiblemente se vieron favorecidas por la oxigenación del agua promovida por el rompimiento de las olas sobre la barrera arrecifal, así como por la presencia de grandes praderas de pastos marinos. En contraste, en el SLN la prolongada residencia de sus aguas (de 2 a 3 años, excepto cuando se presentan fenómenos meteorológicos mayores, como el huracán Wilma en octubre de 2005) y mayor contenido de biomasa del zooplancton promovieron valores ligeramente menores de oxígeno disuelto (Cuadro 1).

El SLN registró la mayor concentración de clorofilaa así como de productividad (biomasa) plánctónica $(0.60$ $\mathrm{mg} / 1$ y $7.5 \mathrm{~g} / 100 \mathrm{~m}^{3}$ respectivamente); la primera estuvo asociada al periodo de mayor precipitación pluvial con una baja salinidad y la segunda, con la época de sequía (Cuadro 1).

Se recolectó un total de 10570225 org./100m ${ }^{3}$ con un promedio mensual de 880835 org./100m $\mathrm{m}^{3}$. Las mayores concentraciones de zooplancteres se presentaron en el SLN (7 022641 org./100m ${ }^{3}$ ) o durante la época de sequía (7 030698 org./100m 3 );se obtuvieron las menores capturas,

Cuadro 1. Variación promedio de las variables superficiales bióticas y abióticas por zona y época en la zona norte del estado de Quintana Roo (2004). \pm Error estandar de la media

\begin{tabular}{|c|c|c|c|c|c|}
\hline \multirow{2}{*}{$\begin{array}{l}\text { División } \\
\text { Variable }\end{array}$} & \multirow[b]{2}{*}{ Costera } & \multicolumn{2}{|l|}{ Zona } & \multicolumn{2}{|c|}{ Época } \\
\hline & & Lagunar & Arrecifal & Sequía & Lluvias \\
\hline Profundidad (m) & $7.7 \pm 1.2$ & $2.5 \pm 0.3$ & $3.6 \pm 0.5$ & $4.0 \pm 0.5$ & $3.9 \pm 0.5$ \\
\hline Temperatura $\left({ }^{\circ} \mathrm{C}\right)$ & $27.4 \pm 0.2$ & $28.2 \pm 0.0$ & $28.4 \pm 0.0$ & $26.4 \pm 0.6$ & $30.6 \pm 0.3$ \\
\hline Salinidad (ups) & $35.8 \pm 0.2$ & $30.4 \pm 1.4$ & $36.1 \pm 0.0$ & $34.2 \pm 0.4$ & $34.1 \pm 0.7$ \\
\hline Ox. disuelto (mg/l) & $4.6 \pm 0.2$ & $4.9 \pm 0.0$ & $5.4 \pm 0.0$ & $5.0 \pm 0.2$ & $5.2 \pm 0.2$ \\
\hline pH (No.) & $7.8 \pm 0.0$ & $7.9 \pm 0.0$ & $7.9 \pm 0.0$ & $8.1 \pm 0.0$ & $7.5 \pm 0.0$ \\
\hline Biomasa $\left(\mathrm{g} / 100 \mathrm{~m}^{3}\right)$ & $3.4 \pm 0.3$ & $7.5 \pm 1.4$ & $3.6 \pm 0.4$ & $5.1 \pm 0.7$ & $4.6 \pm 0.8$ \\
\hline Clorofila- $a$ (mg/l) & $0.25 \pm 0.0$ & $0.69 \pm 0.1$ & $0.18 \pm 0.0$ & $0.33 \pm 0.0$ & $0.40 \pm 0.1$ \\
\hline
\end{tabular}


ya fuera en la zona costera o en la época de lluvias (Cuadro 2). Cada una de las zonas de muestreo, con respecto a las otras, fue significativamente diferente (ANOVA ${ }_{\mathrm{F} 2,810=5.00 \text {, }}$ $\mathrm{P} \leq 0.05$ ), en términos de la abundancia de los grupos de zooplancteres.

El zooplancton recolectado estuvo representado por 41 grupos, de los cuales los copépodos (61\%), las larvas de equinodermos $(17 \%)$ y las larvas de decápodos (5\%), fueron los más importantes, sumando más del $82 \%$ de la fauna recolectada (Cuadro 2). Otros grupos abundantes fueron gasterópodos, medusas, quetognatos (principalmente Ferosagitta hispida Conant), las larvas ofiopluteus de equinodermos, larváceos (Oikopleura sp.) o protozoarios foraminíferos (Globigerina $\mathrm{sp}$.).

En general, los copépodos fueron el grupo más abundante, tanto en el SLN como en la zona costera adyacente; en particular el copépodo Acartia tonsa Dana dominó en el SLN, mientras que las especies más abundantes en la zona costera adyacente fueron Acartia spinata Esterly, Pseudocalanus sp, Calanopia americana Dahl, y en ocasiones Farranula Gracilis Dana.

En particular, los equinodermos fueron abundantes en el SLN; en ocasiones se capturaron numerosas larvas del tipo ofiopluteus (ofiuridos) y en menor cantidad equinopluteus (erizos de mar). Las larvas auricularia, bipinaria, y braquiolaria fueron escasas y se capturaron principalmente en las estaciones del medio marino adyacente. Los foraminíferos estuvieron representados por Globigerina sp, la cual fue más abundante en el medio arrecifal y escasa en el SLN; sus mayores concentraciones se presentaron durante la época de lluvias (Cuadro 2).

El grupo de los decápodos estuvo principalmente representado en la forma larval de zoeas, que corresponde al grupo taxonómico de los braquiuros. Este grupo fue mayormente abundante en el SLN, donde aparecieron en mayor cantidad durante la época de sequía. De manera similar al grupo anterior, los gasterópodos fueron registrados en abundancias importantes en el SLN durante las lluvias (Cuadro 2).

Las medusas fueron el sexto grupo en abundancia $(1.26 \%)$. Eirene lactea Mayer (Hidromedusae) fue la especie dominante dentro de este grupo de depredadores gelatinosos. Respecto a Scyphozoa, la medusa más representativa fue Linuche unguiculata (Swartz), la cual aparece en grandes números en el Caribe mexicano en forma cíclica, usualmente desde finales de febrero hasta finales de mayo o mediados de junio (Cuadro 2).

Los quetognatos se capturaron por lo general en el SLN (159 194) o durante la temporada de secas (142 174). El grupo estuvo formado por 9 especies de las cuales Ferosagitta hispida fue dominante, en particular dentro de los cuerpos de agua del SLN (estaciones 3 a 5), lugares donde fue la única especie registrada. Quetognatos, como Flaccisagita enflata Grassi , Krohnitta pacifica (Aida) y Serratosagitta serratodentata (Krohn) se capturaron con cierta abundancia y frecuencia en la zona costera adyacente. En tanto que Pterosagitta draco (Krohn) Flaccisagita hexaptera (D’'Orbigny) Sagitta bipunctata (Quoy y Gaimard), Sagitta helenae (Ritter-Zahony y Sagitta tenuis (Conant) fueron escasas y raramente capturadas.

Los huevos y larvas de peces abundaron en la zona arrecifal y durante la época de estiaje (Cuadro 2). Se identificaron 54 familias y 115 taxa de larvas de peces, cuya mayor riqueza estuvo conformada por góbidos (Ctenogobius sp., Gobionellus sp., y Gobiosoma sp.), en particular del SLN, donde fue la familia mejor representada. En la zona costera y arrecifal, otras familias como Clupeidae (Harengula jaguana Poey y Jenkinsia lamprotaenia Gosse), Gobiesocidae (Gobiesox strumosus Cope), y Dactyloscopidae (Gillelus uranoidea Böhlke y G. Jacksoni Dawson) fueron las que se capturaron en mayor número. En el análisis por época del año fueron los góbidos, una vez más, los más abundantes y las mayores capturas totales en la temporada de secas en el SLN.

Finalmente, el análisis ACC entre las variables hidrobiológicas del medio y la abundancia de los principales grupos del zooplancton estableció que la varianza explicada que vincula el componente 1 con el componente 2 fue de $86.9 \%$, indicando una alta relación entre ambos. Asimismo, el análisis anterior permitió registrar la relación de larvas de equinodermos y los quetognatos con la biomasa zooplanctónica y la clorofila a, las medusas y larvas de cirripedios con la temperatura y el oxígeno disuelto; los protozoarios y huevos de peces se relacionaron con la salinidad, mientras que los copépodos mantuvieron relación con casi todas las variables del medio (Fig.2).

\section{Discusión}

Las condiciones hidrológicas de la región costera del norte de Quintana Roo, permitieron la diferenciación de 3 áreas con características propias: la zona costera (cuyos parámetros registrados y fauna plánctica indicaron condiciones oceánicas) conformada por las estaciones 1 y 2; la zona lagunar compuesta por las estaciones 3 a 6 y aquellas estaciones relacionadas con la barrera arrecifal (de la 7 a la 12).

Las mayores temperaturas fueron localizadas en el SLN por ser cuerpos de agua someros, así como en las estaciones asociadas a la barrera arrecifal (también de poca profundidad). De acuerdo con Riley y Chester (1989), es probable que esto traiga como consecuencia un 
Cuadro 2. Variación de la densidad de zooplancteres (org./100 $\mathrm{m}^{3}$ ) por zona y época en la región costera norte de Quintana Roo, durante un ciclo anual (2004)

\begin{tabular}{|c|c|c|c|c|c|c|}
\hline \multirow{2}{*}{$\begin{array}{l}\text { División } \\
\text { Grupos }\end{array}$} & \multicolumn{3}{|c|}{ Zona } & \multicolumn{2}{|c|}{ Época } & \multirow{2}{*}{$\begin{array}{c}\text { Total } \\
\%\end{array}$} \\
\hline & Costera & $S L N$ & Arrecifal & Sequía & Lluvias & \\
\hline Protozoa (Foraminifera) & 36800 & 14513 & 451179 & 322731 & 179761 & 4.75 \\
\hline Medusae & 1783 & 21063 & 110605 & 35593 & 97858 & 1.26 \\
\hline Siphonophora & 25578 & 223 & 6524 & 27190 & 5135 & 0.30 \\
\hline Anthozoa & 421 & 588 & 1181 & 1369 & 821 & 0.02 \\
\hline Ctenophora & 7 & 191 & & 116 & 82 & $<0.01$ \\
\hline Turbellaria & 119 & 596 & 17 & 569 & 163 & $<0.01$ \\
\hline Polychaeta & 4180 & 6667 & 4383 & 11451 & 3779 & 0.14 \\
\hline Bivalvia & 465 & 13729 & 1095 & 11017 & 4272 & 0.14 \\
\hline Gasteropoda & 6971 & 192265 & 19291 & 152767 & 65760 & 2.06 \\
\hline Heteropoda & 157 & & 10 & 167 & & $<0.01$ \\
\hline Pteropoda & 1261 & 538 & 531 & 2122 & 208 & 0.02 \\
\hline Cladocera & 15148 & 2560 & 2028 & 6225 & 13511 & 0.18 \\
\hline Ostracoda & 585 & 11556 & 2884 & 8186 & 6839 & 0.14 \\
\hline Copepoda & 426990 & 4207534 & 1824235 & 4487113 & 1971646 & 61.10 \\
\hline Cirripedia & 2640 & 80734 & 182661 & 72635 & 193400 & 2.51 \\
\hline Stomatopoda & 346 & 21 & & 65 & 302 & $<0.01$ \\
\hline Myscidacea & & & 1082 & 1082 & & 0.01 \\
\hline Amphipoda & 131 & 499 & 453 & 613 & 470 & 0.01 \\
\hline Euphausiacea & 135 & & 111 & 247 & & $<0.01$ \\
\hline Decapoda & 26523 & 428850 & 66477 & 419335 & 102515 & 4.93 \\
\hline Bryozoa & 1626 & 56 & 407 & 1771 & 318 & 0.02 \\
\hline Chaetognatha & 7127 & 159194 & 15827 & 142174 & 39974 & 1.72 \\
\hline Echinodermata & 47718 & 1703626 & 31389 & 1029958 & 752775 & 16.86 \\
\hline Larvacea & 49361 & 59194 & 24329 & 71092 & 61792 & 1.25 \\
\hline Thaliacea & 3474 & 78605 & 175 & 82215 & 39 & 0.77 \\
\hline Ascidias & 635 & 3078 & 1084 & 3156 & 1641 & 0.04 \\
\hline Hemichordata & 221 & 108 & 396 & 349 & 376 & $<0.01$ \\
\hline Invertebrados (Larvas) & 4454 & 724 & 27436 & 31647 & 967 & 0.30 \\
\hline Huevos de pez & 23387 & 18631 & 70370 & 89021 & 23367 & 1.06 \\
\hline Larvas de pez & 1995 & 6040 & 4978 & 9510 & 3503 & 0.12 \\
\hline Isopoda & 81 & 5997 & 2956 & 6219 & 2815 & 0.08 \\
\hline Tanaidacea & 102 & 208 & 373 & 409 & 274 & $<0.01$ \\
\hline Cumacea & 26 & 435 & 125 & 34 & 552 & $<0.01$ \\
\hline Chelicerata & 87 & 128 & 392 & 525 & 82 & $<0.01$ \\
\hline Branchiopoda & 11 & & 93 & 104 & & $<0.01$ \\
\hline
\end{tabular}


Cuadro 2. Continúa

\begin{tabular}{|c|c|c|c|c|c|c|}
\hline \multirow{2}{*}{$\begin{array}{l}\text { División } \\
\text { Grupos }\end{array}$} & \multicolumn{3}{|c|}{ Zona } & \multicolumn{2}{|c|}{ Época } & \multirow{2}{*}{$\begin{array}{c}\text { Total } \\
\%\end{array}$} \\
\hline & Costera & SLN & Arrecifal & Sequía & Lluvias & \\
\hline Phoronidea & 188 & & & 188 & & $<0.01$ \\
\hline Nematoda & 75 & 1037 & 372 & 463 & 1021 & 0.01 \\
\hline Trematoda & & 5 & & & 5 & $<0.01$ \\
\hline Hidrozoa & 41 & 8 & 174 & 223 & & $<0.01$ \\
\hline Insecta (larvas) & & 54 & 18 & 7 & 65 & $<0.01$ \\
\hline Total & 691513 & 7022641 & 2855871 & 7030698 & 3539327 & 100.0 \\
\hline Std & 65387 & 685264 & 284511 & 696889 & 318596 & \\
\hline Riqueza & 38 & 36 & 37 & 40 & 35 & 41 \\
\hline
\end{tabular}

calentamiento más rápido con lo cual se eleva la tasa de evaporación, en especial durante la época veraniega que es más cálida. El abatimiento de la temperatura superficial a principios y finales del año se debe a la presencia de vientos fríos del norte, así como a la descarga de aguas subterráneas continentales, sobretodo en el SLN (BoaGonzález,1993).

La disminución de la salinidad en el SLN es ocasionada por los aportes de agua del subsuelo en particular por aquellos situados en el margen occidental del sistema (Merino et al.,1990), así como por escurrimientos provenientes de la llanura de inundación adyacente a la laguna y de las abundantes lluvias en la región que contribuyen a la dilución de la masa de agua sobre todo hacia el ultimo tercio del año (Boa-González, 1993).

La fisiografía del sistema lagunar y su reducido intercambio de agua con el medio marino adyacente, debido entre otras cosas a una reducida variación de marea, contribuye a una escasa renovación de las aguas propiciando tasas de residencia del orden de años (Merino et al., 1990; Vázquez-Uribe, 1992). Por el contrario, la mayor salinidad en el medio arrecifal se debe a una constante comunicación con el medio oceánico, generando una continua renovación de sus aguas. Los aportes de agua continental subterránea en esta área (ojos de agua), aunque presentes, son rápidamente homogenizados debido a la gran dinámica de las corrientes, por lo que sólo tienen influencia muy puntual en el medio arrecifal, como lo han señalado Merino y Otero (1991).

El contenido de oxígeno superficial en las estaciones asociadas al medio arrecifal se vio favorecido por el mayor oleaje y transparencia , así como por una mayor actividad fotosintética, producto de la presencia de extensas praderas de pastos marinos. En contraste, las aguas de menor transparencia ocasionada por resuspensión de los sedimentos, floraciones de microalgas que favorecieron una mayor abundancia en las poblaciones de zooplancton del SLN generaron valores menores de oxígeno disuelto. Al respecto, Boa-González (1993) señala que los menores valores de oxígeno estuvieron asociados con el margen occidental de la laguna, como consecuencia del aporte de agua continental proveniente del flujo subterráneo y que afloran en la laguna en forma de ojos de agua. En este mismo contexto, Merino et al. (1990) informan que el SLN se encuentra bajo un proceso de eutrofización debido a que en el pasado las aguas residuales provenientes de los asentamientos humanos fueron vertidas, sobre todo, en la laguna Bojórquez. Este material orgánico resuspendido (por la navegación de embarcaciones dedicadas a servicios turísticos) o generalizado como material sedimentado, similarmente requiere de oxígeno para llevar a cabo su transformación en los procesos de oxidación-reducción (Valdés-Lozano et al., 2006). Por lo tanto, es importante considerar que muy posiblemente la dinámica ambiental de cada uno de estos sistemas (lagunar, oceánico y arrecifal) sea el resultado de sus características fisiográficas, tiempo de residencia de sus aguas, aportes epicontinentales, dirección e intensidad de los vientos e influencia de la cercanía del medio oceánico que da condiciones hidrológicas propias a cada sistema, como lo han señalado Merino y Otero (1991), Vázquez-Uribe (1992) y Boa-González (1993). Estas condiciones hidrológicas influyeron, a su vez, sobre la composición y abundancia de la comunidad planctónica y que se ha registrado para otras regiones del mundo (Smetacek,1988).

Las condiciones ya mencionadas, presentes en el 


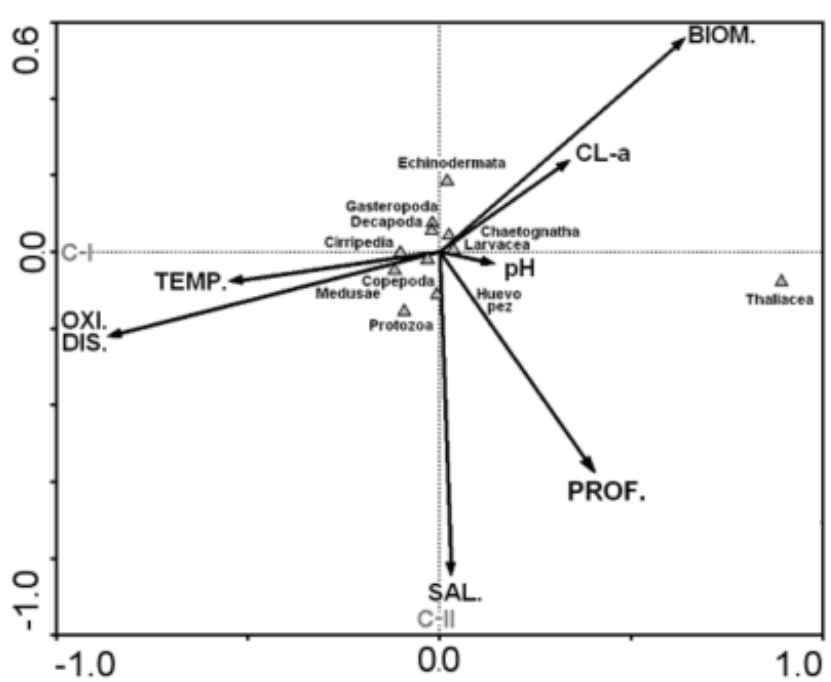

Figura 2. Proyección ortogonal del ACC entre los principales grupos del zooplancton y las variables hidrobiológicas de la zona costera norte de Quintana Roo. Los datos fueron transformados a logaritmo de base 10 .

SLN, generaron una mayor cantidad de clorofila-a y de biomasa zooplánctónica. Es posible que la productividad alta se debiera a importantes aportes de micronutrientes a través del flujo de agua subterránea y a los escurrimientos de la zona de inundación adyacente que son rápidamente aprovechados por el fitoplancton y éste, a su vez, por el zooplancton. Por el contrario, las aguas que rodean los sistemas arrecifales suelen ser menores en productividad planctónica (Sorokin, 1995), lo anterior es corroborado al obtener bajos valores de clorofila-a y biomasa zooplánctica en esta zona. Los cambios en la riqueza y abundancia del zooplancton en este estudio estuvieron relacionados con el medio hidrológico de cada zona y con sus variaciones temporales; cambios que también han sido señalados en otros sistemas costeros en la península y en donde también se ha reconocido la dominancia de los cópepodos con respecto a los otros grupos del zooplancton (ÁlvarezCadena et al., 1996; Álvarez-Cadena y Segura-Puertas, 1997; Suárez-Morales y Rivera-Arriaga, 1998; EscamillaSánchez y Suárez Morales, 2001; Ordóñez-López y Ornelas-Roa, 2003).

La fauna de copépodos para el SNL en este trabajo tiene similitud con la que registran Álvarez-Cadena et al. (1996b), quienes destacan la dominancia de A. tonsa (del orden del 90\%) en la laguna de Bojórquez del SLN. Otros grupos importantes dentro de la citada laguna fueron los de larvas de decápodos y gastrópodos. Para la laguna arrecifal de Puerto Morelos, Álvarez-Cadena et al. (1998) señalan que A. spinata (>67\%) fue la especie más abundante, seguida de Calanopia americana, Clausocalanus furcatus Brady y Subeucalanuus subcrassus Grisbrecht. Estos autores registraron una alta riqueza para el área, 87 especies, que es el número más alto registrado a la fecha, incluso para otros lugares del mismo sistema arrecifal del Caribe de México, como Mahahual (Suárez-Morales y Gasca, 2000). Las diferencias en estos resultados tienen que ver, cuando menos en parte, con la hora de los muestreos (nocturnos), toda vez que muchas de las especies demersales tienden a migrar hacia la superficie en condiciones de oscuridad, como lo han señalado Alldredge y King (1980). Otra posible fuente de disimilitud es el hecho de que Álvarez Cadena et al. (1996) no hicieron muestreo en el SLN, donde $A$. tonsa es el copépodo más abundante, como ya se mencionó.

La composición y variación de la abundancia de las medusas registradas sugiere que la fauna de medusas del norte de Quintana Roo presenta una mezcla de especies neríticas y oceánicas. El trabajo de Muñoz-Salcedo (2005), con material de este mismo estudio, informa que estos organismos estuvieron representados por 33 especies, de las cuales 29 fueron hidromedusas con una dominancia de Eirene lactea, y 3 escifomedusas, siendo la más representativa Linuche unguiculata (Swartz), especie que aparece en forma temporal y cíclica, usualmente de febrero a finales de mayo o inicios de junio. Las grandes concentraciones en que se presenta han sido motivo de varios estudios relacionados con su toxicidad y actividad urticante que ocasiona molestias a los bañistas.

La dominancia de F. hispida en el medio lagunar y de F. enflata (Grassi) en la zona costera han sido señaladas por Ramírez-Ávila y Álvarez-Cadena (1999) para la laguna arrecifal de Puerto Morelos. Así también los resultados en este estudio confirman lo registrado por Álvarez-Cadena et al. (1996) en un trabajo previo donde F. hispida se registra como la única especie para el SLN. El registro se confirma en este estudio, y sus resultados, al parecer, tienen su origen en las características eurihalinas y euritermas de la especie. Sin embargo, es pertinente considerar también lo sugerido por Bieri (1991), quien catalogó este quetognato como casi planctónico, debido a que durante el día tiende a migrar al fondo y adherirse a varios tipos de substrato (macroalgas, pastos marinos, etc). En este sentido el sistema lagunar Nichupté, de poca profundidad, presenta estas características de grandes cantidades de vegetación sumergida, posiblemente como resultado del rápido aprovechamiento en el contenido de nutrientes en el sistema, lo cual da a lugar un rápido crecimiento de la vegetación sumergida (Smith et al., 1981; Barko y Smart, 1982, 1986; Valiela, et al., 1990; Hermus, 1992) y que es aplicable en particular para Bojorquez y las cuencas norte y centro, proporcionado en forma importante grandes camas 
de vegetación sumergida como substrato de adhesión para esta especie.

La riqueza de larvas de peces registrada en esta zona norte (54 familias y 115 taxa) es alta, lo que sugiere que la principal fuente de organismos es la zona arrecifal. Al respecto, Ramírez-Ávila (2001) registró la presencia de 55 familias y 197 especies en el arrecife de Puerto Morelos y la zona marina adyacente, destacando la dominancia de larvas de Ctenogobius sp., Syngnathus floridae Jordan y Gilbert y Jenkinsia lamprotaenia (Gosse). Asimismo, los cambios en la composición y abundancia de las larvas de peces registrados en el presente estudio estuvieron en relación con el medio hidrológico de cada zona y de sus variaciones temporales. Estos cambios también se han señalado en otros sistemas costeros en la península (Vásquez-Yeomans et al.,1998; Vásquez-Yeomans, 2000; Ordóñez-López, en prensa).

La heterogeneidad física del medio ejerce efectos importantes sobre la comunidad (Begon et al., 1999). La relación de algunos zooplancteres con la biomasa zooplanctónica y clorofila denota la estrecha interacción trófica existente entre ellos. Al respecto, sabemos que un incremento en la productividad primaria (fitoplancton) resulta en un aumento en la comunidad del zooplancton, que a su vez constituirá el alimento de otros grupos de consumidores en la red trófica. Lo anterior queda demostrado al obtener en el presente estudio la relación de los quetognatos y la productividad plánctónica. En este sentido, Roff et al. (1988) registraron que los máximos de clorofila y concentración del fitoplancton están relacionados con muchas agrupaciones del zooplancton; por ejemplo, con las etapas de desarrollo de los copépodos y con los estadios nauplios de otros grupos holoplanctónicos, lo cual influye en forma determinante en la distribución de herbívoros.

Las relaciones entre la temperatura y oxígeno disuelto con la abundancia de medusas y larvas de cirripedios presuponen que estos grupos sean tolerantes a valores altos de la temperatura. Al respecto, Loman-Ramos (2005) registró que muchas especies de medusas neríticas y oceánicas se han considerado como euritérmicas. Este mismo autor también indica que las medusas proliferan rápidamente durante los meses más cálidos en aguas costeras, donde la productividad biológica favorece el rápido desarrollo poblacional de sus presas potenciales.

Los datos faunísticos sugieren que la composición general del zooplancton de las zonas costera y arrecifal es bastante similar, pero no necesariamente el del ambiente lagunar del SLN. De la misma manera, diversos trabajos han discutido la relación de los zooplancteres con el medio hidrológico costero; sin embargo, es evidente que la heterogeneidad ambiental e hidrológica no fueron los únicos factores que determinaron los patrones de distribución del zooplancton. Es probable que otros factores físicos (patrón de circulación) y biológicos (flujo de estadios tempranos, disponibilidad de alimento, competencia y depredación) tengan un papel importante en la variación de la abundancia y composición de los organismos que forman la comunidad del zooplancton en la región costera norte de Quintana Roo.

Finalmente, un aspecto que no debe soslayarse es el hecho de que la parte norte del Caribe mexicano es un área donde los asentamientos humanos se han incrementado en forma substancial. Toda la región comprendida desde Cancún, Puerto Morelos, Playa del Carmen hasta Tulum, presentan en la actualidad un gran desarrollo de infraestructura turística. Todo ello tiene un costo biológico-ambiental, y los arrecifes coralinos, que son uno de los principales atractivos para el turismo en el área, son organismos delicados en extremo que ciertamente se ven afectados por el incremento de las actividades humanas (por ejemplo, el contacto directo, o el desecho de compuestos contaminantes, tales como gasolinas o aceites de los motores de las lanchas). Esto sin contar eventos meteorológicos catastróficos, como lo fue el huracán Wilma en 2005, o los ya consabidos efectos del calentamiento global, lo que lleva a proponer que los estudios a mediano y largo plazo deban promoverse y mantenerse,en lo posible, pues finalmente son éstos los que darán mas información e indicaciones mejor fundamentadas del estado de salud o de deterioro del ambiente arrecifal.

\section{Agradecimientos}

A Lourdes Segura-Puertas y a Laura Celis-Gutiérrez por su colaboración en el análisis de los grupos de zooplancton, y a Yazmín Ramírez Ávila y Margarita Ornelas-Roa por la identificación de las larvas de peces.

\section{Literatura citada}

Aldredge, A. y J. M. King. 1977. Distribution, abundance and substrate preferences of demersal reef zooplankton at Lizard Island Lagoon, Great Barrier Reef. Marine Biology. 41:317-333.

Aldredge, A. y J. M. King. 1980. Effects of moonlight on the vertical migration patterns of demersal plankton. Journal of Experimental Marine Biology and Ecology 44:13-56.

Álvarez-Cadena, J.N., M. E. Islas-Landeros y E. SuárezMorales. 1996b. A preliminary zooplankton survey in a Mexican Caribbean eutrophic coastal lagoon. 
Bulletin of Marine Science 58:694-708.

Álvarez-Cadena, J. N. y L. Segura-Puertas.1997. Zooplankton variability and copepod assemblages from a tropical coastal lagoon. Gulf. Research Reports 9:345-355.

Álvarez-Cadena, J. N., E. Suárez-Morales y R. Gasca. 1998. Copepod assemblages from a reef- related environment in the Mexican Caribbean Sea. Crustaceana 71:411-433.

Álvarez-Cadena, J. N., E. Suárez-Morales y J. A. Mclelland.1996. Observations on the population dynamics of Sagitta hispida Conant (Chaetognatha) in a tropical lagoon system of northeast Yucatan (Mexican Caribbean). Gulf Research Reports 9:197-204.

Barko, J.W. y R. M. Smart. 1982. Mobilization of sediment phosphorous by submersed freshwater macrophytes. Freshwater Biology 10:229-238.

Barko, J.W. y R. M. Smart. 1986. Sediment related mechanisms of growth limitation in submersed macrophytes. Ecology 67:1328-1340.

Beers, J. R. 1981. Determinación de la biomasa del zooplancton. In Atlas del zooplancton del Atlántico sudoccidental y métodos de trabajo con el zooplancton marino, D. Bolstovskoy (ed.). Instituto Nacional de Investigación y Desarrollo Pesquero (INIDEP), Mar del Plata. p. 133-141.

Begon, M., Harper, J. y C. Towsend. 1999. Ecology: individuals, populations and communities, tercera edición. Blackwell Science, London. 1148 p.

Bieri, R. 1991. Six new genera in the chaetognaths family Sagittidae. Gulf Research Reports 8:221-225.

Boa-González, J. 1993. Estudio de calidad del agua del sistema lagunar Nichupté en Cancún, Quintana Roo. Informe Interno. Comisión Nacional del Agua, Gerencia Regional Sureste, Mérida, Yucatán. 55 p.

Escamilla-Sánchez, J. y E. Suárez-Morales. 2001. Distribución del zooplancton durante flujos de marea opuestos en el complejo lagunar de Chelem, Yucatán. Revista de Biología Tropical 49:47-51.

Harris, R.P., P.H. Wiebe, J. Lenz, H. R. Skojdal y M. Huntley. 2000. Zooplankton Methodology Manual. Academic, London. 684 p.

Hermus, K. 1992. Production and morphology of Thalassia testudinum König,in relation with severalenvironmental parameters in the northern part of Nichupté Lagoon System, Mexico. Tesis maestría Neederlands Instituut Oecologisch Onderzoek. Centrum voor Estuariene en Mariene Oecologie, Yerseke. $61 \mathrm{p}$

Instituto de Geofísica. 1991..Tablas de predicción de mareas. 1992. Puertos del golfo de México y mar Caribe. Servicio Mareográfico Nacional. Instituto de Geografía, Universidad Nacional Autónoma de
México, México, D.F. 189 p.

Loman-Ramos, L. 2005. Medusas del sur del golfo de México: variación espacial en la estructura comunitaria y asociaciones durante el otoño de 1999. Tesis maestría Biología Marina, Instituto Politécnico Nacional, Centro de Investigación y Estudios Avanzados, Unidad Mérida, Departamento de Recursos del Mar, Yucatán. 75 p.

Merino, M. 1986. Aspectos de la circulación costera superficial del Caribe mexicano con base en observaciones utilizando tarjetas de deriva. Anales del Instituto de Ciencias del Mar y Limnología, Universidad Nacional Autónoma de México 13:31-46.

Merino M., S. Czitrom, E. Jordan, E. Martín, P. Thomé y O. Moreno.1990. Hydrology and rain flushing of the Nichupté lagoon system, Cancún, Mexico. Estuarine, Coastal and Shelf Science 30:223-237.

Merino, M. y L. Otero. 1991. Atlas ambiental costero de Puerto Morelos, Quintana Roo. Centro de Investigaciones de Quintana Roo, Chetumal. 80 p.

Muñoz-Salcedo, G. 2005. Distribución espacio-temporal de la fauna de medusas en la zona costera del norte de Quintana Roo, México. Informe final de servicio social. Universidad Autónoma Metropolitana, Unidad Xochimilco, México, D.F. 24 p.

Ordóñez-López, U. Dinámica temporal del ictioplancton asociado al arrecife de Puerto Morelos, Quintana Roo: taxonomía, estructura de la comunidad y su relación con el medio. Tesis doctorado, Instituto de Ciencias del Mar y Limnología. Universidad Nacional Autónoma de México. 268 p. (en prensa).

Ordóñez- López, U. y M. Ornelas-Roa. 2003. Variaciones de la comunidad de copépodos pláncticos en el gradiente estuarino-costero de Celestún, Yucatán, México. Hidrobiológica 13:231-238.

Ramírez-Ávila, Y. 2001. Ictioplancton arrecifal frente a Puerto Morelos, Quintana Roo, durante la época de lluvias: patrón a fina escala de la estructura de la comunidad, ensamblages y su relación con el medio. Tesis de maestría Instituto Politécnico Nacional, Centro de Investigación y Estudios Avanzados, Unidad Mérida, Yucatán. 107 p.

Ramírez-Ávila, Y. y J. N. Álvarez-Cadena. 1999. Chaetognath species composition from a coral reef lagoon in the Mexican Caribbean Sea. Revista de Biología Tropical 47(supl. 1):157-163.

Ramos-Viera, G. 2001. Cnidarios pláncticos (Medusae) de la laguna arrecifal de Puerto Morelos, Quintana Roo. Tesis, Universidad Autónoma de Baja California Sur, Área Interdisciplinaria de Ciencias Marinas, Departamento de Biología Marina. 51 p.

Riley, J. P. y R. Chester. 1989. Introduction to Marine 
Chemistry. Academic, London. 459 p.

Roff, J.C., K. Middlebrook y F. Evans. 1988. Longterm variability in North Sea zooplankton off the Northumberland coast: productivity of small copepods and analysis of trophic interactions. Journal of the Marine Biological Association of the United Kingdom 68:143-164.

Smetacek, V. 1988. Plankton characteristics. In Ecosystems of the world. Continental shelves, H. Postma and J. Zijlstra (eds.). Elsevier, New York. p. 93-130.

Smith, S.V., W.J. Kimmerer, E. A. Laws, R.E. Brock y T. W. Walsh. 1981. Kaneohe Bay sewage diversion experiment: perspectives on ecosystem responses to nutritional perturbation. Pacific Science 35:278-596.

Sorokin, Y. I. 1978. Microbial production in coral reef community. Archives of Hydrobiology 83:281-323.

Sorokin, Y. I. 1995. Coral reef ecology. Springer, Berlin. $465 \mathrm{p}$.

Striclan, J.D.H. y T.R. Parson. 1972. A practical handbook of sea water analysis. Bulletin of Fisheries Research Board, Ottawa 122 p.

Suárez-Morales, E. y R. Gasca. 1990. Variación dial del zooplancton asociado a praderas de Thalassia testudinum en una laguna arrecifal del mar Caribe mexicano. Universidad y Ciencia 7:141-146.

Suárez-Morales, E. y R. Gasca. 2000. The planktonic copepod community at Mahahual reef, Western Caribbean. Bulletin of Marine Science 66:255-267.
Suárez-Morales, E. y E. Rivera-Arriaga. 1998. Zooplancton e hidrodinámica en zonas litorales y arrecifales de Quintana Roo, México. Hidrobiológica 8:19-32.

Ter Braak, C.J.F. y P. F. M. Verdonschot. 1995. Canonical correspondence analysis and related multivariate methods in aquatic ecology. Aquatic Science 37:255289.

Valdés-Lozano, D. S., M. Chumacero y E. Real. 2006. Sediment oxygen consumption in a developed coastal lagoon of the Mexican Caribbean. Indian Journal of Marine Science 35:227-234.

Valiela, I., J. Costa, K. Foreman, J. M. Teal, B. Howes y D. Aubrey. 1990. Transport of ground water-borne nutrients from watershed and their effects on coastal waters. Biogeochemist 10:77-197.

Vásquez-Yeomans, L. 2000. Seasonal variation of ichthyoplankton in a western Caribbean bay system. Environmental Biology of Fish 58:349-392.

Vásquez-Yeomans, L., U. Ordóñez-López y E. SosaCordero. 1998. Fish larvae adjacent to a coral reef in the western Caribbean Sea off Mahahual, Mexico. Bulletin of Marine Science 62:229-245.

Vázquez-Uribe, J. 1992. Estudio hidrodinámico de la laguna de Nichupté, Cancún, Quintana Roo. Segundo informe ADISA e ICA. Proyectos Especializados de Ingeniería. México, D.F. 30 p.

Zar, J. H. 1984. Biostatistical analysis. Prentice Hall, Englewood Cliffs, New Jersey. 620 p. 